\title{
Pathological findings in a patient with non- dystrophic myotonia with a mutation of the SCN4A gene; a case report
}

Takanori Hata, Takamura Nagasaka* ${ }^{*}$, Kishin Koh, Mai Tsuchiya, Yuta Ichinose, Haitian Nan, Kazumasa Shindo and

Yoshihisa Takiyama

\begin{abstract}
Background: Non-dystrophic myotonias (NDMs) are skeletal muscle disorders involving myotonia distinct from myotonic dystrophy. It has been reported that the muscle pathology is usually normal or comprises mild myopathic changes in NDMs. We describe various pathological findings mimicking those of myotonic dystrophy (DM) in biopsied muscle specimens from a patient with NDMs with a long disease duration.

Case presentation: A 66-year-old Japanease man presented eye closure myotonia, percussion myotonia and grip myotonia together with the warm-up phenomenon and cold aggravation from early childhood. On genetic analysis, a heterozygous mutation of the SCN4A gene (c.2065 C > T, p.L689F), with no mutation of the CLCN1, DMPK, or ZNF9/CNBP gene, was detected. He was diagnosed as having NDMs. A biopsy of the biceps brachii muscle showed increasing fiber size variation, internal nuclei, chained nuclei, necrotic fibers, fiber splitting, endomysial fibrosis, pyknotic nuclear clumps and disorganized intermyofibrillar networks. Sarcoplasmic masses, tubular aggregates and ragged-red fibers were absent.

Conclusion: It is noteworthy that the present study revealed various pathological findings resembling those seen in DM, although the pathology is usually normal or mild in NDMs. The pathological similarities may be due to muscular modification with long-standing myotonia or excessive muscle contraction based on abnormal channel activity.
\end{abstract}

Keywords: Non-dystrophic myotonia, Myotonic dystrophy, Muscle biopsy, SCN4A

\section{Background}

Non-dystrophic myotonias (NDMs) are skeletal muscle disorders involving myotonia distinct from myotonic dystrophy caused by a mutation of the DMPK or ZNF9/ $C N B P$ gene. NDMs comprise a heterogeneous group of neuromuscular disorders caused by mutations of skeletal muscle chloride channels (CLCN1) and sodium channels (SCN4A), and include myotonia congenita, paramyotonia congenital (PMC), potassium-aggravated myotonia or sodium channel myotonia, and hyperkalemic periodic paralysis (HYPP) [1-3]. In 1991, Ptacek et al. [1] reported a linkage of PMC with the SCN4A gene, and suggested that PMC and HYPP are allelic disorders.

\footnotetext{
* Correspondence: nagat@yamanashi.ac.jp

Department of Neurology, Faculty of Medicine, University of Yamanashi,

1110, Shimokato, Chuou-city, Yamanashi 409-3898, Japan
}

Meanwhile, myotonic dystrophy type 2 (DM2), a multisystemic disease manifesting cataracts, grip myotonia, proximal limb muscle weakness, tremors, etc., can exhibit an almost pure myotonic phenotype and is difficult to clinically differentiate from NDMs [3]. The CLCN1 and $S C N 4 A$ mutations have been reported as modifying factors for DM2 $[4,5]$.

NDMs are diagnosed based on symptoms, electromyography and muscle pathology before categorization based on genetic analysis. It has been reported that the muscle pathology is usually normal or comprises mild myopathic changes in NDMs [6-9].

We describe various pathological findings mimicking those of DM in biopsied muscle specimens from a patient with NDMs with a long disease duration.

(c) The Author(s). 2019 Open Access This article is distributed under the terms of the Creative Commons Attribution 4.0 International License (http://creativecommons.org/licenses/by/4.0/), which permits unrestricted use, distribution, and reproduction in any medium, provided you give appropriate credit to the original author(s) and the source, provide a link to the Creative Commons license, and indicate if changes were made. The Creative Commons Public Domain Dedication waiver (http://creativecommons.org/publicdomain/zero/1.0/) applies to the data made available in this article, unless otherwise stated. 


\section{Case presentation}

A 66-year-old Japanese man complained of increasing muscle stiffness of the four extremities and difficulty in relaxing his grip, which had been present from early childhood. His parents were consanguineous and his family had no neuromuscular disease (Fig. 1a). In his youth, mild muscle weakness and muscular hypertrophy had developed. Later in his life, gait disturbance due to muscle stiffness, severe in the starting period and relieved on continuation of walking, developed. The patient was diagnosed as having myotonia congenita at the age of 22 without any complications (Fig. 1b). He was treated with acetazolamide and phenytoin with relief of the symptoms. In spite of the symptoms, his daily activities were almost normal including working as a gardener, except that his myotonic symptoms got worse at low temperatures. There was no paralytic event during the entire disease course. At the age of 62 , his muscular symptoms worsened on discontinuation of phenytoin due to membranous nephropathy, so he was admitted to our hospital. On admission, he had a Hercules-like appearance with hypertrophy of the limbs and axial muscles including the pectoralis major muscles. Physical examination revealed eye closure myotonia, percussion myotonia and grip myotonia. The myotonia improved with muscle exercise or repeated effort, the so-called "warm-up phenomenon", and was aggravated by exposure to cold. Ocular movement was restricted in every direction with relief on repetition. Dysphagia and dysarthria were also present only when he was exposed to cold. Muscle manual testing was normal at ordinary temperatures.

The other neurologic findings were all normal. There had been no paralytic event or systemic abnormality. Laboratory examination showed an increased level of serum creatine kinase, $388 \mathrm{U} / \mathrm{l}$ (normal value < 279 U/l), which decreased with rest in hospital. Electrocardiography, ultrasound cardiography and chest X-raying were normal. Needle electromyography of the left biceps brachii muscle and quadriceps femoris muscle showed

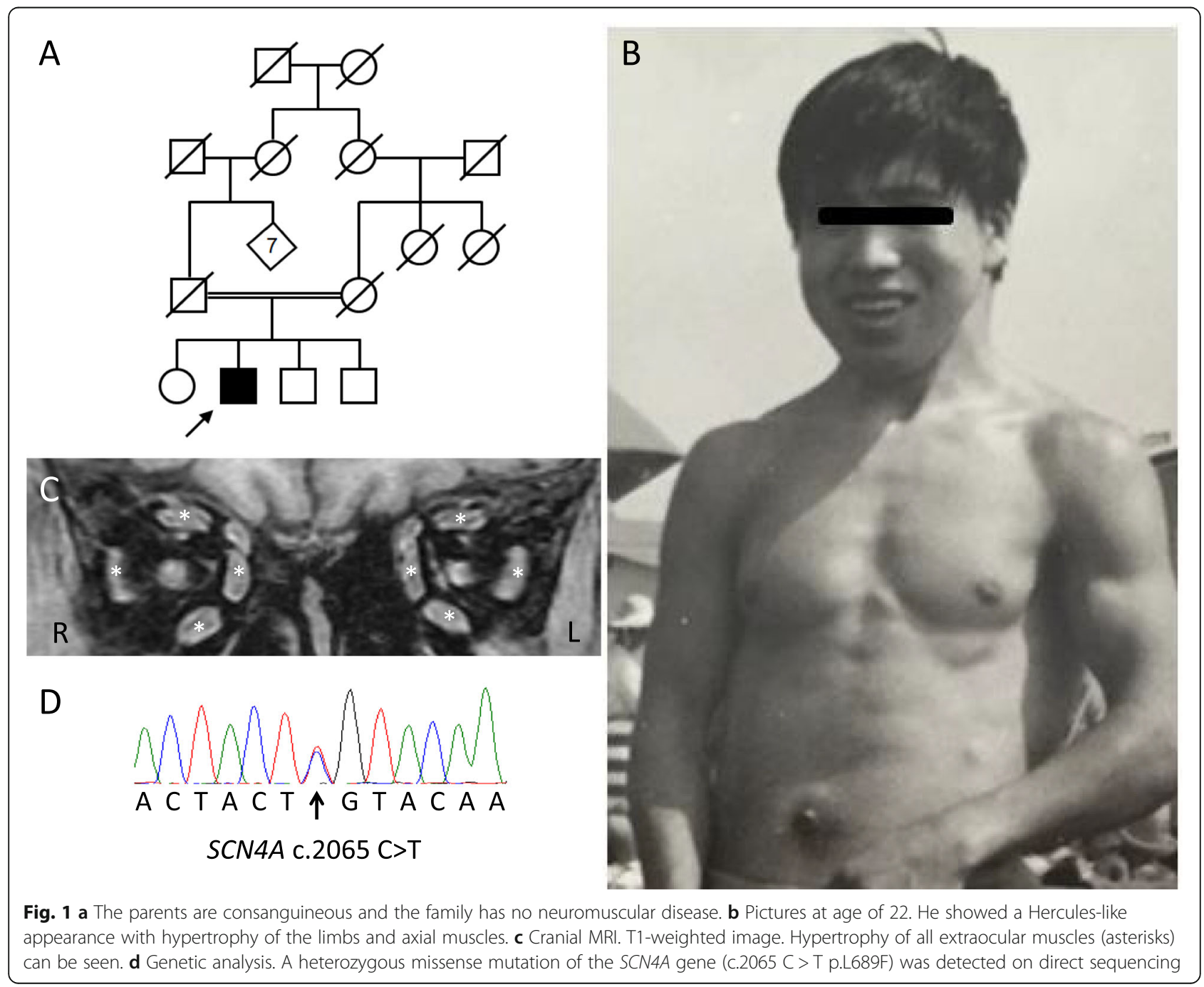


myotonic discharges (Additional file 1). T1-weighted images of Cranial MRI revealed hypertrophy of all extraocular muscles (Fig. 1c). His myotonic symptoms improved since the start of administration of mexiletine (300 mg/day).
Genetic analysis: Whole-exome sequencing revealed a heterozygous mutation of the SCN4A gene (c.2065 $\mathrm{C}>\mathrm{T}$, p.Leu689Phe). However, we found no mutation of the CLCN1 gene. Then, we confirmed the SCN4A mutation by direct sequencing (Fig. 1d). Mutation
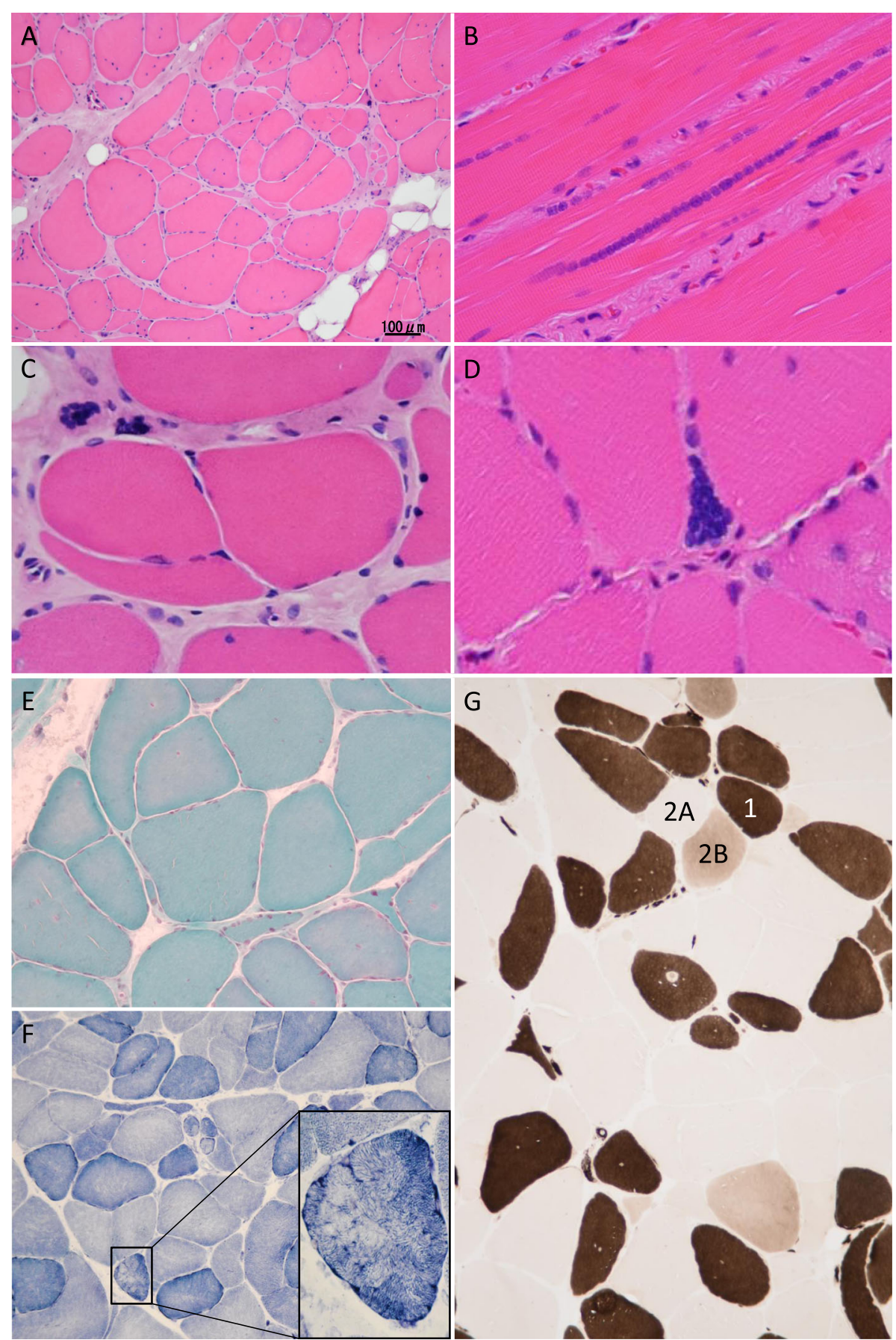

Fig. 2 a-d HE staining. Variation in diameter of muscle fibers including hypertrophy (over 100 um) and atrophy was observed (a). Internal nuclei, chained nuclei (b), fiber splitting (c), pyknotic nuclear clumps (d), endomysial fibrosis and mild fatty replacement were observed in all areas. e modified Gomori trichrome staining. Ragged-red fibers could not be observed but fibers exhibiting slight marginal hyperstaining were observed. f NADH-TR( $\beta$ nicotinamide adenine dinucleotide tetrazolium reductase)staining. Disorganized intermyofibrillar networks including a moth-eaten appearance and lobulated fibers could be seen. $\mathbf{g}$ Myosin ATPase staining. Reduction of type 2B fibers and predominancy of type 2A fibers could be seen 
analysis showed no mutation of the DMPK or ZNF9/ CNBP gene.

\section{Muscle biopsy}

A muscle biopsy was performed on the right biceps brachii muscle, followed by light microscopy and routine electron microscopy. On HE staining, variation in diameter of muscle fibers including hypertrophy (over $100 \mu \mathrm{m}$, about a half of muscle fibers) and atrophy was observed (Fig. 2a, Additional files 2 and 3). Internal nuclei, chained nuclei (Fig. 2B), fiber splitting (Fig. 2c), pyknotic nuclear clumps (Fig. 2d), endomysial fibrosis and mild fatty replacement were observed in all areas. Sarcoplasmic masses were absent. On modified Gomori trichrome staining (mGT), ragged-red fibers could not be observed but fibers exhibiting slight marginal hyperstaining were observed (Fig. 2e). NADH-TR staining showed disorganized intermyofibrillar networks including a motheaten appearance and lobulated fibers (Fig. 2f). Myosin ATPase staining showed reduction of type $2 \mathrm{~B}$ fibers and predominancy of type 2A fibers (Fig. $2 \mathrm{~g}$ ). On electron microscopy, atrophic fibers exhibiting Z-streaming were observed (Additional file 2). Tubular aggregates were absent in all examinations.

\section{Discussion and conclusion}

In the past, NDMs were classified into three genetic categories, i.e., dominant or recessive $C L C N 1$ mutations, and dominant SCN4A ones. However, it has been clarified that myotonias with these mutations have various clinical phenotypes [3, 10-12].

Irrespective of causative channel mutations, the muscle pathologies of NDMs are limited to mild nonspecific myopathic changes [6]. There is no major pathological qualitative difference between mutated $S C N 4 A$ and mutated $C L C N 1$ muscle. Mild variation of fiber size and type 2 predominancy with a reduction or absence of type $2 \mathrm{~B}$ fibers were commonly noted in several cases with mutated $S C N 4 A[7,8]$, and mutated CLCN1 $[9,13$, 14]. Other findings, including centrally located nuclei [8] and excessive mitochondria without aggregation in NDMs [15-17], an increase in endomysium connective tissue in CLCN1 [18], and tubular aggregates and vacuoles in myotonia congenita [19] have been reported. There were few findings supportive of light microscopic findings as to ultrastructural changes $[19,20]$. In the present case, different from the above findings, the muscle pathology closely resembled that in DM, as summarized in Table 1 [21, 22]. Increased internal nuclei, pyknotic nuclear clumps, chained nuclei and fiber splitting are the same findings as in DM. Other findings including variation of fiber diameter, endomysial fibrosis, fiber type predominancy and the type of atrophy differ between DM and our case. In summary, major differences in our case compared with DM are as follows, the dystrophic changes are not so severe, and regeneration of muscle fibers is superior to degeneration. The reason why the pathological changes in NDMs were stated to be mild in the past reports may be that the examinations were performed in childhood or adolescence. As in the present case, pathological changes similar to those in DM might be due to the long term myotonia or excessive muscle contraction, although the mechanism of muscle degeneration is likely not the same for the two diseases.

Schoser et al. [20] proposed that sodium channelopathies may mimic the phenotypic features of DM2 in older patients based on the muscle pathology in patients with a heterozygous mutation of the SCN4A gene (A1481D). Although the pathological features of NDMs are essentially different from DM1 and DM2 [21], see Table 1., DM2 is clinically the most important disorder

Table 1 Comparison of pathological findings between DM1, DM2 and the present case

\begin{tabular}{|c|c|c|c|c|}
\hline & Present case & DM1 & DM2 & NDMs \\
\hline Fiber size variation & ++ & +++ & +++ & + \\
\hline Endomysial fibrosis & + & +++ & ++ & \pm \\
\hline Increase of internal nuclei & +++ & +++ & +++ & \pm \\
\hline Pyknotic nuclear clumps & + & + & +++ & - \\
\hline Sarcoplasmic masses & - & ++ & \pm & - \\
\hline Chained nuclei & + & + & unknown & - \\
\hline Fiber splitting & + & + & unknown & - \\
\hline Ragged red fibers & - & + & \pm & - \\
\hline Fiber type predominancy & $2 \mathrm{~A}>1>>2 \mathrm{~B}$ & type1 & type1 & $2 \mathrm{~A}>1>>2 \mathrm{~B}$ \\
\hline Type of atrophy & nonspecific & type 1 > type2 & type2 & type2B \\
\hline Ring fibers & - & ++ & \pm & - \\
\hline Fatty replacement & + & +++ & ++ & - \\
\hline
\end{tabular}

+++ severe; ++ modest; + mild; \pm occasionally present; - absent 
for differential diagnosis of NDMs in adulthood. It is because DM2 has mild muscular involvement and mild or few systemic complications [23]. So we analyzed $D M P K$ and $C N B P$ for differential diagnosis, and confirmed no mutation.

Genetic screening of $S C N 4 A$ in our case revealed a heterozygous missense mutation (c.2065C > T, p.Leu689Phe). The mutation was reported only in one family i.e., the proband and her daughter, as a de novo mutation [24]. The proband of the family showed a Thomsen-like phenotype without muscle weakness like in the present case.

In daily practice, the diagnosis of NDMs or DM is based on clinical findings and on genetic testing, and a muscle biopsy in the patients is dispensable. A muscle biopsy in patients with myotonia is significant in terms of a better understanding of the pathophysiology. We need further examination for associating the described pathological findings with SCN4A-related NDMs.

\section{Additional files}

Additional file 1: Figure S1. Needle electromyography. Myotonic discharges could be seen for the left biceps brachii muscle and quadriceps femoris. (PPTX $194 \mathrm{~kb}$ )

Additional file 2: Figure S2. Electron microscopic findings. Atrophic fibers exhibiting Z-streaming were observed. (PPTX $321 \mathrm{~kb}$ )

Additional file 3: Figure S3. Diameter of muscle fibers. Muscle fiber diameter was determined for 234 fibers in cross-section. The average diameter is $100.6 \mu \mathrm{m}$, ranging from 6.8 to 118.2 . $48.7 \%$ of muscle fibers exhibit hypertrophy (over $100 \mu \mathrm{m}$ ). (PPTX $46 \mathrm{~kb}$ )

\section{Abbreviations}

ATPase: Adenosine triphosphatase; DM: Myotonic dystrophy; HE: Hematoxylin-eosin; HYPP: Hyperkalemic periodic paralysis; mGT: Modified Gomori trichrome staining; MRI: Magnetic resonance imaging; NADH-TR: $\beta$ nicotinamide adenine dinucleotide tetrazolium reductase; NDMs: Nondystrophic myotonias; PMC: Paramyotonia congenital

\section{Acknowledgments}

Not applicable.

\section{Authors' contributions}

TH and TN mainly drafted the manuscript and Figs. TH and MT mainly provided the clinical information including medical history, neurological findings, electrophysiological evaluation and treatment. TH and TN analyzed and interpreted the pathological findings. $\mathrm{KK}, \mathrm{YI}$ and $\mathrm{HN}$ acquired, analysed and interpreted the genetic data. KS and YT supervised the entire work. All authors read and approved the contents of the case report.

\section{Funding}

We received no specific funding for this work.

\section{Availability of data and materials}

All data generated or analysed during this study are included in this published article.

\section{Ethics approval and consent to participate}

This case report involves a patient. The case report complies with the Helsinki Declaration, and approval was obtained from the ethics committee of the University of Yamanashi, Faculty of Medicine.

\section{Consent for publication}

Written informed consent was obtained from the patient for publication of this case report including the pedigree diagram and any other associated images.

\section{Competing interests}

The authors declare that they are no competing interests.

Received: 4 October 2018 Accepted: 6 June 2019

Published online: 12 June 2019

\section{References}

1. Ptacek LJ, Trimmer JS, Agnew WS, Roberts JW, Petajan JH, Leppert M. Paramyotonia congenita and hyperkalemic periodic paralysis map to the same sodium-channel gene locus. Am J Hum Genet. 1991;49:851-4.

2. Cannon SC. Pathomechanisms in channelopathies of skeletal muscle and brain. Annu Rev Neurosci. 2006:29:387-415.

3. Trivedi JR, Bundy B, Statland J, Salajegheh M, Rayan DR, Venance SL, et al. Non-dystrophic myotonia: prospective study of objective and patient reported outcomes. Brain. 2013;136:2189-200.

4. Suominen T, Schoser B, Raheem O, Auvinen S, Walter M, Krahe R, et al. High frequency of co-segregating CLCN1 mutations among myotonic dystrophy type 2 patients from Finland and Germany. J Neurol. 2008;255:1731-6.

5. Bugiardini E, Rivolta I, Binda A, Soriano Caminero A, Cirillo F, Cinti A, et al. SCN4A mutation as a modifying factor of myotonic dystrophy type 2 phenotype. Neuromuscul Disord. 2015;25:301-7.

6. Fisher ER, Danowski TS, Ahmad U, Breslau P, Nolan S, Stephan T. Electron microscopical study of a family with myotonia congenita. Arch Pathol. 1975; 99:607-10.

7. Kubota T, Kinoshita M, Sasaki R, Aoike F, Takahashi MP, Sakoda S, et al. New mutation of the $\mathrm{Na}$ channel in the severe form of potassium-aggravated myotonia. Muscle Nerve. 2009;39:666-73.

8. Borg K, Ahlberg G, Anvret M. C4342T-mutation in the SCN4A gene on chromosome $17 \mathrm{q}$ in a Swedish family with paramyotonia congenita (Eulenburg) - correlations with clinical, neurophysiological and muscle biopsy data. Neuromuscul Disord. 1997:7:231-3.

9. Ulzi G, Lecchi M, Sansone V, Redaelli E, Corti E, Saccomanno D, et al. Myotonia congenita: novel mutations in the CLCN1 gene and functional characterizations in Italian patients. J Neurol Sci. 2012;318:65-71.

10. Dupré N, Chrestian N, Bouchard JP, Rossignol E, Brunet D, Sternberg D, et al. Clinical, electrophysiologic and genetic study of non-dystrophic myotonia in French-Canadians. Neuromuscul Disord. 2009;19:330-4.

11. Matthews E, Fialho D, Tan SV, Venance SL, Cannon SC, Sternberg D, et al. The non-dystrophic myotonias: molecular pathogenesis, diagnosis and treatment. Brain. 2010;133:9-22

12. Ptacek $L$, Johnson KJ, Griggs RC. Genetics and physiology of the myotonic muscle disorders. N Engl J Med. 1993;328:482-9.

13. Portaro S, Altamura C, Licata N, Camerino GM, Imbrici P, Musumeci O, et al. Clinical, molecular, and functional characterization of CLCN1 mutations in three families with recessive myotonia congenita. NeuroMolecular Med. 2015;17:285-96

14. Gurgel-Giannetti J, Senkevics AS, Zilbersztajn-Gotlieb D, Yamamoto LU, Muniz VP, Pavanello R, et al. Thomsen or Becker myotonia? A novel autosomal recessive nonsense mutation in the CLCN1 gene associated with a mild phenotype. Muscle Nerve. 2012;45:279-83.

15. Gay S, Dupuis D, Faivre L, Masurel-Paulet A, Labenne M, Colombani M, et al. Severe neonatal non-dystrophic myotonia secondary to a novel mutation of the voltage-gated sodium channel (SCN4A) gene. Am J Med Genet A. 2008; 146:380-3.

16. Crews J, Kaiser KK, Brooke MH. Muscle pathology of myotonia congenita. J Neurol Sci. 1976;28:449-57.

17. Heene R, Gabriel R-R, Manz F, Schimrigk K. Type 2B muscle fibre deficiency in myotonia and paramyotonia congenita a genetically determined histochemical fibre type pattern? J Neurol Sci. 1986;73:23-30.

18. Hilbert $P$, Frank $S$, Raheem $O$, Suominen T, Penttilä S, Udd B, et al. Normal muscle MRI does not preclude increased connective tissue in muscle of recessive myotonia congenita. Acta Neurol Scand. 2011;124:146-7.

19. Schröder JM, Becker PE. Alterations of the T-system and the sarcoplasmic reticulum in myotonia, paramyotonia and adynamia. Virchows Arch A Pathol Pathol Anat. 1971;357:319-44. 
20. Schoser BG, Schröder JM, Grimm T, Sternberg D, Kress W. A large German kindred with cold-aggravated myotonia and a heterozygous A1481D mutation in the SCN4A gene. Muscle Nerve. 2007;35:599-606.

21. Vihola A, Bassez G, Meola G, Zhang S, Haapasalo H, Paetau A, et al. Histopathological differences of myotonic dystrophy type 1 (DM1) and PROMM/DM2. Neurology. 2003;60:1854-7.

22. Meola G. Clinical aspects, molecular pathomechanisms and management of myotonic dystrophies. Acta Myol. 2013;32:154-65.

23. Meola G, Cardani R. Myotonic dystrophy type 2: an update on clinical aspects, genetic and Pathomolecular mechanism. J Neuromuscul Dis. 2015; 2:S59-71.

24. Trip J, Drost G, Verbove DJ, van der Kooi AJ, Kuks JB, Notermans NC, et al. In tandem analysis of $\mathrm{CLCN1}$ and $\mathrm{SCN} 4 \mathrm{~A}$ greatly enhanced mutation detection in families with non-dystrophic myotonia. Eur J Human Genet. 2008;16:921-9.

\section{Publisher's Note}

Springer Nature remains neutral with regard to jurisdictional claims in published maps and institutional affiliations.

Ready to submit your research? Choose BMC and benefit from:

- fast, convenient online submission

- thorough peer review by experienced researchers in your field

- rapid publication on acceptance

- support for research data, including large and complex data types

- gold Open Access which fosters wider collaboration and increased citations

- maximum visibility for your research: over $100 \mathrm{M}$ website views per year

At BMC, research is always in progress.

Learn more biomedcentral.com/submissions 